\title{
A Simplified Protocol for Apoptosis Assay by DNA Content Analysis
}

\author{
Ruoxiang Wang ${ }^{1}$ and Yu-Fang Shi ${ }^{2}$ \\ ${ }^{1}$ Department of Urology, Emory University School of Medicine, Atlanta, GA, and 2 Department of Molecular Ge- \\ netics, Microbiology and Immunology, University of Medicine and Dentistry of New Jersey-Robert Wood Johnson \\ Medical School, Piscataway, NJ, USA
}

BioTechniques 33:S88-S91 (October 2002)

\begin{abstract}
Apoptotic cells possess specific morphological and biochemical markers. Various methods have been developed to detect apoptosis based on these markers. One of the most common is the fragmentation of genomic DNA. In addition to electrophoresis for the identification of the characteristic 200 base pair ladders and terminal deoxyribonucleotidyltransferase-mediated dUTP-biotin nick end-labeling, DNA content analysis is often employed. This technique is based on the fact that permeablized apoptotic cells release fragmented DNA, resulting in DNA content that is less than that in live diploid cells. Although widely used, we have found that the number of apoptotic cells detected by DNA content analysis is often lower than that detected by other methods. We have developed a simplified version of the flow cytometry-based protocol that detects a number of apoptotic cells closer to that detected by other methods, and which requires a dramatically reduced number of cells. In addition, this simplified protocol allows preparation of a large number of samples at the same time.
\end{abstract}

\section{INTRODUCTION}

Apoptosis is the major form of programmed cell death, in which a doomed cell performs suicidal self-destruction under the direction of its own genetic program (6). Mechanisms responsible for apoptosis have been suggested to be associated with activation of a protease cascade, which results in hydrolysis of vital cellular structures, including nucleosomal degradation of the genomic DNA (3). Apoptosis research has been incorporated into virtually every aspect of biological studies and, correspondingly, technologies for sensitive and quantitative analysis of apoptosis have been developed.

In the past decade, a plethora of methods has been adapted for in vivo and in vitro detection of apoptosis (2). These methods are based on various aspects of the biochemical changes in the apoptosis process. Among them, the most convincing proof of apoptosis is the detection of the characteristic loss of genomic material in the affected cells. Whereas the terminal deoxyribonucleotidyltransferase mediated dUTP-biotin nick end labeling
(TUNEL) assay is the current popular method for detection of apoptosis in situ, and whereas electrophoretic fractionation of nucleosomal genomic DNA is frequently the choice in other situations, flow cytometry is now a major method for apoptosis detection (7). Genomic DNA in the nuclei of apoptotic cells is often fragmented. The fragments are released upon cell membrane permeablization. Thus, after staining with DNA intercalating fluorescent dye (propidium iodide), nuclei with lost genomic material are shown in a subdiploidal peak in a conventional cell cycle analysis by flow cytometry. Due to its quantitative nature, this flow cytometry analysis could provide accurate information about the percentage of cells that have undergone apoptosis.

The technology of apoptosis detection by flow cytometry was derived from cell cycle analysis (1). Corresponding protocols were adapted from the conventional flow cytometric analysis of apoptotic cells. Conventionally, cell samples for apoptosis studies are collected in single cell suspension. Following centrifugation, cell pellets are washed in isotonic buffer. The samples are then fixed in ethanol, which is later removed by further centrifugation and washing. The sample is then treated by ribonuclease and stained by propidium iodide. The conventional protocol is laborious, requiring a fair amount of hands-on time. This is especially cumbersome when a large number of samples are to be analyzed. Moreover, we have noticed marked discrepancies in the ratio of apoptotic events in the same sample determined by different methods. For later stages of cell death, for example, the apoptosis ratio determined by flow cytometry is frequently much lower than that by trypan blue staining (used for a later stage of cell death) and by other methods. This suggests that flow cytometry analysis of apoptosis could give distorted results, which is likely due to loss of dead cells in the process of sample preparation.

Here, we describe a simplified protocol for preparation of samples for assessing apoptosis by DNA content analysis. This protocol omits several steps from the original protocol and requires fewer cells in each sample. We present results to prove that this simplified protocol provides a more sensitive and convenient method for detecting apoptosis. 


\section{MATERIALS AND METHODS}

\section{Cell Culture}

Human peripheral white blood cells were obtained from buffy coat preparations with the Ficoll ${ }^{\circledR}$-Plaque gradient separation. Murine thymocytes and splenocytes were prepared from 8week-old male Balb/c mice and cultured under published conditions (5). The method for the maintenance of murine $\mathrm{T}$ lymphoid hybridoma cell line A1.1 has been described (4).

\section{Reagents}

Cell culture media was purchased from GIBCO/Invitrogen (Grand Island, NY, USA) and Ficoll-Plaque was from Pharmacia (Peapack, NJ, USA). Antibody to murine T cell receptor (anti-CD3) was obtained from Pharmingen (La Jolla, CA, USA). All other chemical reagents, including propidium iodide and trypan blue, were purchased from Sigma (St. Louis, MO, USA) in the purest form available.

\section{Induction of Apoptosis}

Apoptosis of the experimental cells was carried out as follows. Murine splenocytes $(1 \times 105 /$ well in a 96-well plate $)$ were treated with $100 \mathrm{nM}$ staurosporine. Murine thymocytes $(1 \times$ $105 /$ well in a 96-well plate) were treated with $1 \mu \mathrm{M}$ dexamethasone. Murine T lymphoid hybridoma A1.1 cells $\left(1 \times 10^{5} /\right.$ well in 96-well plate) were activated by anti-CD3 coated plates. All the treatments were for 16 hours.
A

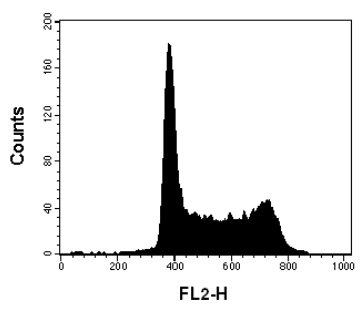

C

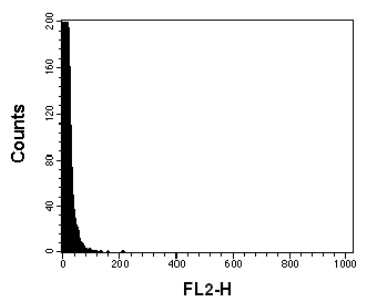

B

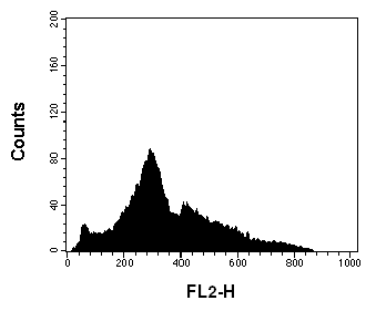

D

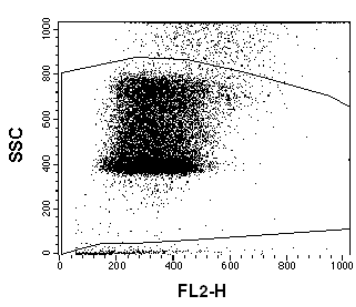

Figure 1. The simplified protocol yields quality samples for flow cytometric assay of apoptosis. T cell hybridoma A1.1 cells were treated with (B) or without (A) plastic bound anti-CD3 for 16 hours. Cells were harvested. The samples were analyzed by a flow cytometer upon PI staining. Unstained cells (C) were used as controls to set the gating (D) for data collection.
Preparation of Samples for Flow Cytometric Analysis of Apoptosis

After induction of apoptosis, samples were collected and resuspended in $20 \mu \mathrm{L}$ of phosphate-buffered saline (PBS). The suspended sample was fixed by direct addition of $80 \mu \mathrm{L}$ of absolute ethanol. The sample was then kept at $4^{\circ} \mathrm{C}$ from $20 \mathrm{~min}$ utes to several months. Before flow cytometric analysis, $400 \mu \mathrm{L}$ of staining buffer $(50 \mu \mathrm{g} / \mathrm{mL}$ of propidium iodide in PBS with freshly added RNase A to $10 \mu \mathrm{g} / \mathrm{mL}$ ) was added to the sample. Following incubation at room temperature for 30 minutes, the sample was directly subjected to flow cytometry analysis.

\section{Flow Cytometry Instruments}

Three generations of the flow cytometry instruments, FACScan $^{\mathrm{TM}}$, FACSCaliber ${ }^{\mathrm{TM}}$, and FACSQuant ${ }^{\mathrm{TM}}$ (Becton Dickinson, San Jose, CA, USA), have been used for validation of the simplified method for detection of apoptosis. For all instruments, the fluorescence of propidium iodide stained DNA was analyzed for FL2 or FL3 on a linear scale.

\section{RESULTS AND DISCUSSION}

The purpose of our modification of the sample preparation protocol for flow cytometric analysis of apoptosis is to establish a procedure that could accurately detect cells containing subdiploid genomic DNA with minimal manipulations of the samples to facilitate simultaneous preparation of multiple samples. The modified protocol reduces the time needed for preparation by $75 \%$ by omitting all the washing steps and many centrifugations during sample preparation.

\section{The Simplified Protocol Yields Quality Samples for Flow Cytometric Assay of Apoptosis}

With this protocol, experimental samples are resuspended in a small volume and fixed by directly adding ethanol to the cell suspension. The resulting fixed samples are in a small volume (about $100 \mu \mathrm{L})$. Samples at this stage can be stored at $4^{\circ} \mathrm{C}$ for several months without affecting the end result (data not shown). This feature is beneficial for kinetic analysis of the apoptosis process, in which many samples have to be collected periodically during a given time span. Before flow cytometry analysis, samples are stained simply by adding four volumes of a staining buffer with ribonuclease for removal of RNA. With the simplified method, we could routinely prepare multiple samples for flow cytometric analysis of apoptotic cells with relatively little effort.

We validated the quality of samples prepared with this simplified protocol. In one example (Figure 1), T cell hybridoma A1.1 cells were induced to undergo $T$ cell receptor activation-induced cell death by cross linking CD3 (4), and $1 \times 10^{5}$ cells were prepared with the simplified method for DNA content analysis. We found that samples prepared with the simplified protocol are of high quality, as shown by a proper cell cycle profile in the control sample (Figure 1A), and a marked subdiploid peak detected in sample treated with anti-CD3 (Figure 1B). To set a proper gating for data collection, cells without propidium iodide stain- 
ing were analyzed on a flow cytometer (Figure $1 \mathrm{C}$ ). This sample provided a standard for gating out DNA-free cell debris. In fact, when a PI-stained sample was analyzed on a dot plot with side scatter on the Y-axis and FL2 (the intensity of DNA intercalated propidium iodide) on the $\mathrm{Y}$-axis, counts outside the gate are mostly leftmost when plotted as a histogram. Therefore, these counts represented cell debris that did not contain genomic DNA and were excluded during data acquisition.

\section{The Simplified Protocol Improves Sensitivity to Detect Apoptotic Events}

To appreciate the superiority of the simplified protocol to the conventional procedure, we compared their sensitivities in detecting apoptotic cells. Following induction of apoptosis, each sample was aliquoted for apoptotic analysis with different methods. We have determined that, for the A1.1 cell line, T-cell re- ceptor activation-induced cell death leads to typical apoptosis in more than $90 \%$ of the population, as shown by microscopic observation and the genomic DNA fragmentation assay by electrophoresis (data not shown) as well as by trypan blue exclusion (Figure 2A). When the same sample was prepared with the conventional protocol, a lower percentage of apoptotic events was obtained (Figure 2B). Nonetheless, samples prepared with the simplified protocol provided a ratio of apoptosis that is comparable to that determined by trypan blue exclusion (Figure 2C). Our data thus indicate that the simplified protocol described here not only shortens the experimental procedure but also increases the sensitivity of detecting apoptosis.

The loss of sensitivity seen in the conventional protocol may be attributed to extensive washings of the experimental samples. Thus, we examined the effect of isotonic washing on detection of apoptotic events. An apoptotic sample was prepared according to the conventional protocol; an aliquot of the sample from each
A

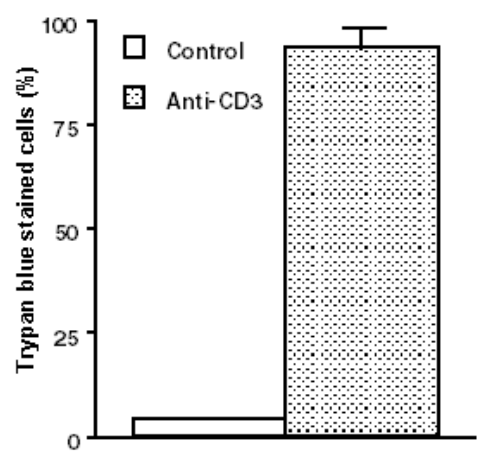

B

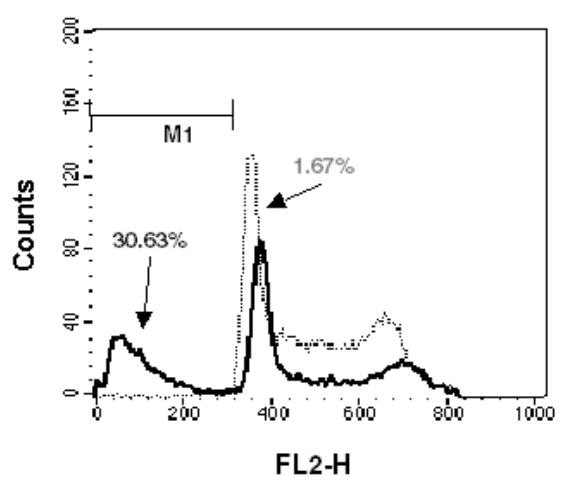

C

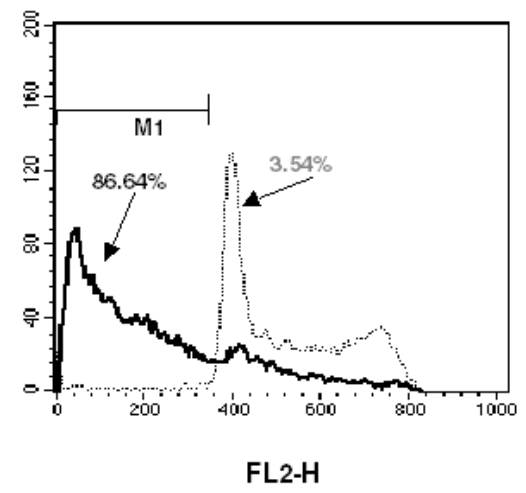

Figure 2. Improved sensitivity to detect apoptotic events. T cell hybridoma A1.1 cells were activated with plastic bound anti-CD3 for 16 hours. Apoptosis was analyzed by microscopic counting of trypan blue stained cells (A), by flow cytometry analysis with conventional sample preparation method (B), and by flow cytometry analysis with the simplified method (C). In panels B and C, solid lines and dotted lines represent anti-CD3 activated samples and control samples, respectively. Values denote percentage of subhaploidal counts in each sample.

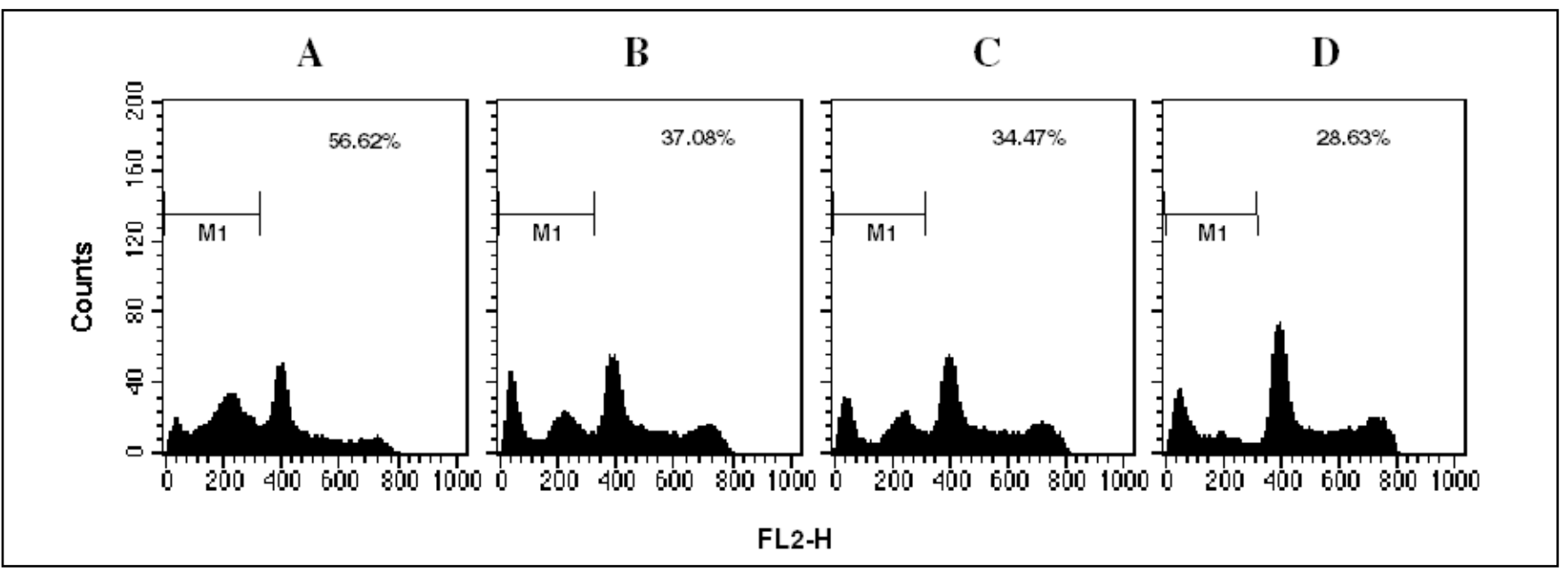

Figure 3. The effect of washing steps on the subdiploid peak. For this experiment, T cell hybridoma A1.1 cells were activated with plastic bound anti-CD3 for 12 hours. Flow cytometric samples were prepared with the simplified method (A), with one washing (B), with two washings (C), and with three washings (D). Values denote percentage of subhaploidal counts in each sample. 
washing was stained and analyzed by flow cytometry (Figure 3). The subhaploidal peak, which reflects the apoptotic events, decreased as the number of washing steps increased, suggesting that genomic fragments in apoptotic cells are washed away.

\section{The Simplified Protocol Requires Fewer Cells in Each Sample}

The simplified protocol described here may avoid unnecessary loss of sample material. This would be an asset to apoptotic analysis of samples with limited availability, or use of the same

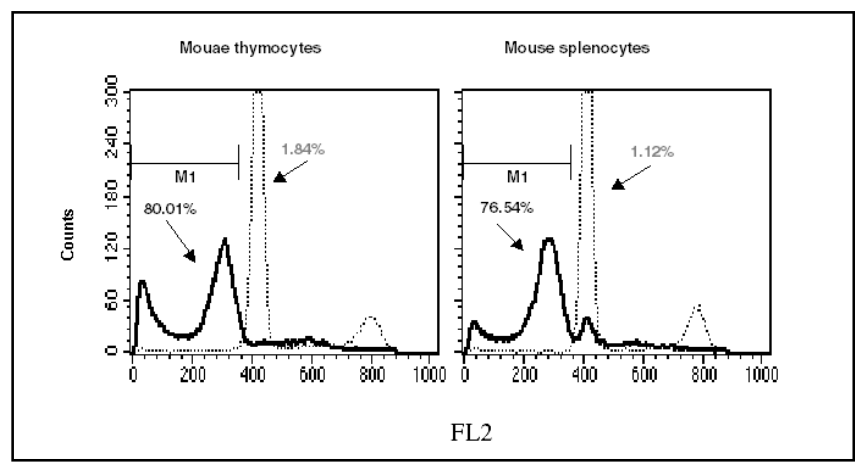

Figure 4. The simplified protocol could be applied to detect apoptosis in primary thymocyte and splenocyte cultures. Splenocytes were treated with staurosporine $(50 \mu \mathrm{M})$ and thymocytes were treated with dexamethasone $(100 \mathrm{nM})$ for 12 hours. Apoptosis was analyzed by the simplified method. Solid lines represent treated samples and dotted lines control samples.

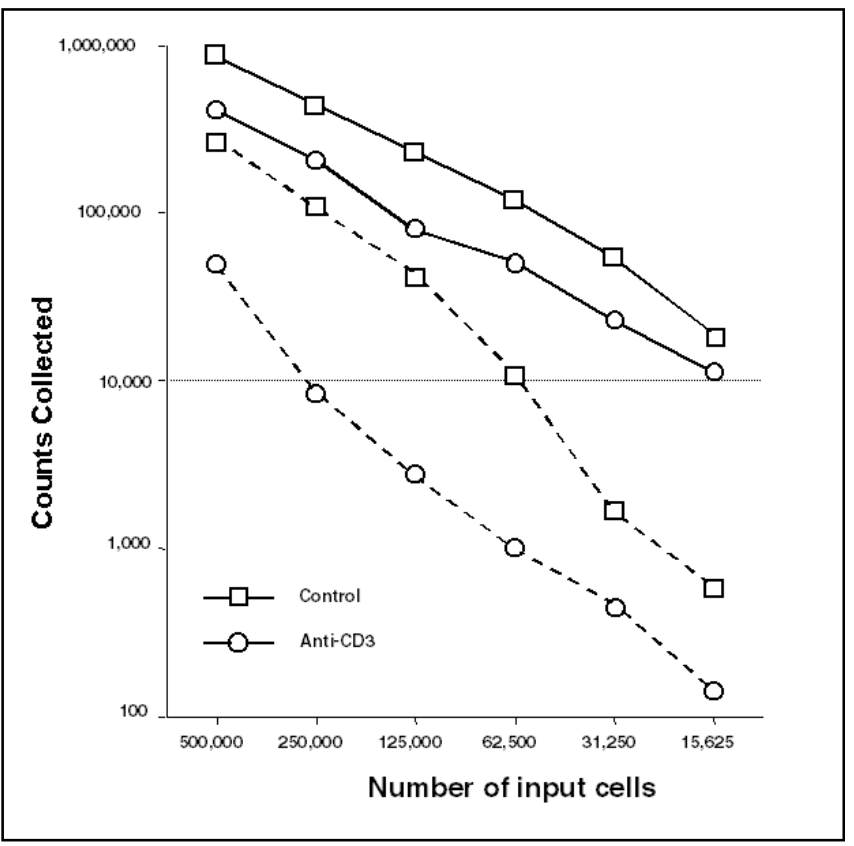

Figure 5. The simplified protocol requires fewer cells in each sample. Different numbers of T cell hybridoma A1.1 cells were treated with or without plastic bound anti-CD3 for 16 hours. To analyze for apoptosis by flow cytometry, cells were collected from each treatment and prepared separately with the simplified (solid lines) and conventional (dotted lines) protocols. Data acquisition was set to $1 \times 10^{6}$ counts to ensure that all the cells in each sample were collected. The total count in each sample was plotted. The fine doted line was used to mark the usual data size (10 000 counts) in a routine analysis of apoptosis by flow cytometry. For each data points, standard deviation was less than $5 \%$ of the mean $(n=3)$. apoptotic sample for multiple analyses. To explore this possibility, we induced apoptosis in $5 \times 10^{5}$ of murine thymocytes or splenocytes. Samples were recovered by centrifugation and pellets resuspended in $30 \mu \mathrm{L}$ of supernatant. Samples were then fixed and stained by the simplified method and subjected to flow cytometry for apoptosis detection (Figure 4). Our data showed that, with the simplified protocol, a fraction of cells for a standard apoptosis experiment would be enough for flow cytometry. In addition, a sample from a standard apoptosis experiment could be used for multiple assays.

Finally, we estimated the minimal cell number required for each sample. For this series of experiments, different numbers of A1.1 cells were subjected to T-cell receptor activation-induced cell death. Duplicate samples were prepared separately with the simplified and the conventional protocols. During flow cytometry, data acquisition was set to $1 \times 10^{6}$ to collect all cells in each sample. We found that while conventional protocol required at least $2.5 \times 10^{5}$ cells for a $1 \times 10^{4}$ data acquisition, the new protocol needs only $1.5 \times 10^{4}$ starting cells for an acquisition of the same sized data pool (Figure 5). Therefore, our protocol only requires one-tenth the number of cells for a DNA content analysis than previous procedures.

\section{REFERENCES}

1.Krishan, A. 1975. Rapid flow cytofluorometric analysis of mammalian cell cycle by propidium iodide staining. J. Cell. Biol. 66:188-193.

2.Loo, D.T. and J.R. Rillema. 1998. Measurement of cell death. Methods Cell Biol. 57:251-264.

3.Sharma, K., R.X. Wang, L.Y. Zhang, D.L. Yin, X.Y. Luo, J.C. Solomon, R.F. Jiang, K. Markos, W. Davidson, D.W. Scott, and Y.F. Shi. 2000. Death the Fas way: regulation and pathophysiology of CD95 and its ligand. Pharmacol. Ther. 88:333-347.

4.Shi, Y.F., M.G. Szalay, L. Paskar, B.M. Sahai, M. Boyer, B. Singh, and D.R. Green. 1990. Activation-induced cell death in T cell hybridomas is due to apoptosis. Morphologic aspects and DNA fragmentation. J. Immunol. 144:3326-3333.

5.Wang, R., L. Zhang, X. Zhang, J. Moreno, C. Celluzzi, M. Tondravi, and Y. Shi. 2002. Regulation of activation-induced receptor activator of NF-kappaB ligand (RANKL) expression in T cells. Eur. J. Immunol. 32:1090-1098. 6. Wyllie, A.H. 1997. Apoptosis: an overview. Br. Med. Bull. 53:451-465.

7.Zamai, L., E. Falcieri, G. Zauli, A. Cataldi, and M. Vitale. 1993. Optimal detection of apoptosis by flow cytometry depends on cell morphology. $\mathrm{Cy}$ tometry 14:891-897.

\section{Address correspondence to:}

Dr. Yu-Fang Shi

Department of Molecular Genetics, Microbiology and Immunology

Robert Wood Johnson Medical School

661 Hoes Lane

Piscataway, NJ 08854, USA

e-mail: shiyu@umdnj.edu 